\title{
Anatomy of the coronary arteries in transposition of the great arteries and methods for their transfer in anatomical correction
}

\author{
MAGDI H YACOUB AND ROSEMARY RADLEY-SMITH \\ From Harefield Hospital, Middlesex, and the National Heart Hospital, London, UK
}

\begin{abstract}
Yacoub, M I, and Radley-Smith, R (1978). Thorax, 33, 418-424. Anatomy of the coronary arteries in transposition of the great arteries and methods for their transfer in anatomical correction. For the success of anatomical correction of transposition of the great arteries (TGA) it is essential to transfer the coronary ostia to the posterior vessel without undue tension, torsion, or kinking of the proximal coronary arteries or their early branches. This requires thorough understanding of the different modes of origin and early branching of the coronary arteries in TGA. Based on observations made during anatomical correction, a classification of the coronary arteries in TGA is suggested. In type $A$ the right and left coronary ostia arise from the middle of the right and left posterior aortic sinuses and curve forwards to reach the right atrioventricular groove or anterior interventricular groove respectively. In type B both coronary arteries arise by a single ostium, while in type $C$ the two coronary ostia are situated posteriorly, very close to each other, in a position similar to that in type B. The origin of the coronary arteries in type D is similar to that of type A. However, the right coronary artery gives origin to the circumflex coronary artery that curves round the posterior (pulmonary) vessel to reach the atrioventricular groove. In type $\mathrm{E}$ the right coronary artery arises in common with the left anterior descending artery from the left posterior sinus, while the circumflex artery arises separately from the right posterior sinus. Three techniques for the transfer of the different types of coronary arteries during anatomical correction are described.
\end{abstract}

Although anatomical correction of transposition of the great arteries (TGA) is the logical and obvious method for treatment of this condition, its successful application has been delayed until very recently (Jatene et al, 1976; Ross et al, 1976; Yacoub et al, 1976). This delay is partly due to the technical difficulties of transferring the coronary ostia, because these vary in position and course and are closely related to the aortic valve. For the success of the operation it is essential to avoid undue tension, torsion, or kinking of the main coronary arteries or their early branches. This can be achieved only by a thorough understanding of the different modes of origin, early course, and branching of the coronary arteries in TGA. We shall attempt to define the different anatomical types of coronary arteries in TGA and to describe methods for transfer of each type.

\section{Material and methods}

The anatomy of the coronary arteries was examined during operation for TGA both before and, whenever possible, after transection of the aorta in 18 patients in an attempt to characterise: (1) The location of each coronary ostium and its relation to the sinuses and commissures of the aortic and pulmonary valves.

(2) The course, direction, and length of the main coronary artery before branches are given off.

(3) The mode of origin and course of the early branches.

ANATOMICAL TYPES OF CORONARY ARTERIES IN TGA Using the criteria mentioned above, the following anatomical types could be identified. 


\section{Type A}

Type A was encountered in 11 patients. The right and left coronary ostia are situated in the centre of the right and left posterior aortic sinuses of Valsalva and are related to the right and left anterior pulmonary sinuses respectively (fig 1a). The most proximal part of the right coronary artery passes horizontally to the right for 2 to $3 \mathrm{~mm}$ before curving forward to reach the right atrioventricular (AV) groove, which it follows as in hearts with normally related great arteries. Just before reaching the AV groove the right coronary artery gives off the conus branch, which passes in front of the anterior ventricular outflow, and the sinus node artery, which runs on the medial aspect of the base of the right atrial appendage towards the junction between the superior vena cava and the right atrium. The first part of the left coronary artery passes horizontally to the left before curving forwards towards the top of the anterior interventricular groove where it divides into the anterior descending and circumflex branches. The left main coronary artery is about 3 to $5 \mathrm{~mm}$ in length and gives off no branches before dividing.

\section{Type $B$}

Type B was encountered in two patients. In this type, both coronary arteries arise by a common ostium in close relation to the posterior aortic commissure (and anterior pulmonary commissure) from either the right or left posterior aortic sinus (fig 1b). The common coronary trunk is usually very short (1-2 $\mathrm{mm})$ and passes almost directly backwards before dividing into the right and left coronary arteries that diverge, passing horizontally between the aorta and pulmonary artery before curving forwards towards the right AV groove for the right coronary artery and the top of the anterior interventricular groove for the left. The mode of branching is usually similar to that in type A. Occasionally, however, the circumflex arises from the right coronary artery and curves around the posterior aspect of the pulmonary artery to reach the posterior $\mathrm{AV}$ groove, as in type D.

\section{Type $C$}

Type $\mathrm{C}$ was encountered in one patient. The two coronary ostia are situated very close to each other in close proximity to the posterior aortic commissure (fig 1c). The initial course of each coronary artery is directly backwards for a very short distance (1-2 mm), before curving sharply to the right or left, respectively. The further course and modes of branching are identical to those of type B

\section{Type D}

The location of the ostia in type $D$ is similar to that of type A-that is, in the middle of the right and left posterior sinuses. The right coronary artery passes horizontally to the right for about 1 to $2 \mathrm{~mm}$ before giving a large circumflex branch that passes almost directly backwards in close relation to the right side of the pulmonary artery, and curves along its posterior aspect to reach the posterior interventricular groove (fig 1d). The left coronary artery has an identical course to that described for type A, except for the absence of a circumflex branch. On reaching the top of the
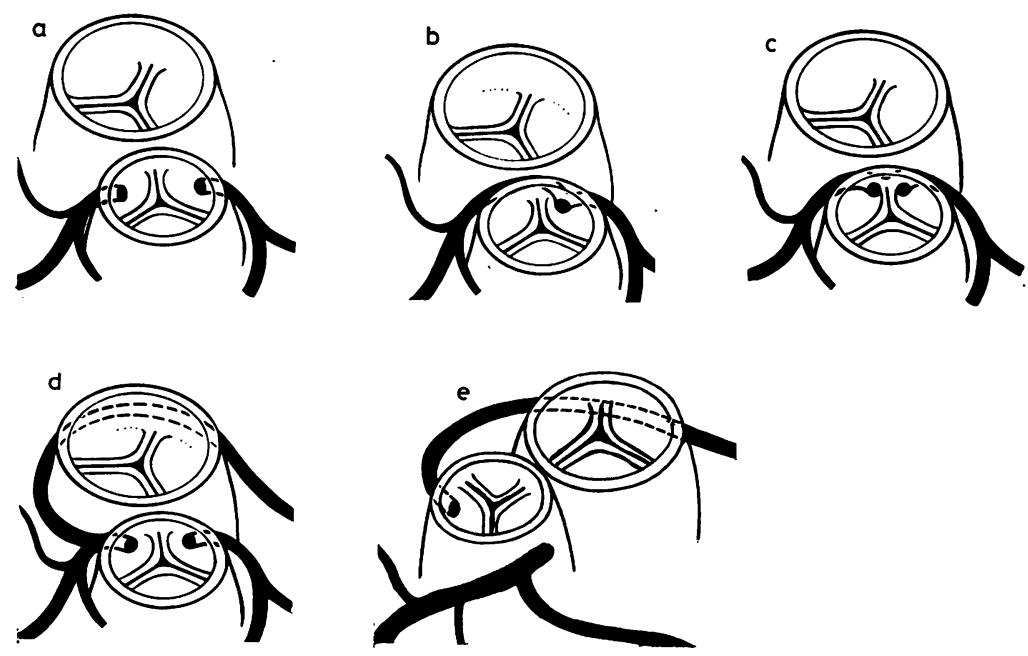

Fig 1 (a) to (e) Five different types of coronary anatomy in TGA (see text). 
anterior interventricular groove, the vessel continues as the anterior descending artery. This type was encountered in three patients.

\section{Type E}

Type $\mathrm{E}$ appears to be a rare type that was encountered in one patient in this series. The circumflex artery arises from the right posterior sinus and its further course is identical to that described in type $D$. Another coronary trunk arises from the anterior part of the left posterior sinus and passes forward for $2 \mathrm{~mm}$ before dividing into the right main and left anterior descending arteries (fig 1e). The right coronary artery passes in front of the outflow tract of the anterior ventricle to reach the right atrioventricular groove. This type was associated with a different relationship of the great arteries that were almost side by side, with the aorta to the right and slightly anterior. In addition, the mode of origin of the aorta from the anterior ventricle was unusual, arising from a recess to the right of the outflow tract, which ended blindly. The relation of the aortic valve to the tricuspid was similar to that encountered in double outlet right ventricle with d-malposition of the great arteries.

\section{TECHNIQUES FOR CORONARY TRANSFER}

Three techniques were evolved for transfer of the different anatomical types of coronary arteries.

\section{TRANSFER OF TYPE A CORONARY ARTERIES}

Before transecting either of the great vessels, it is important to identify two points on the posterior vessel for future positioning of the coronary ostia without kinking. These points are marked by two 6/0 sutures placed in the adventitia of the posterior vessel (fig 2). After it has been confirmed that the pulmonary valve and left ventricular outflow are normal, both great arteries are transected about $3 \mathrm{~mm}$ above the top of the aortic sinuses of Valsalva (fig 3 ). The coronary ostia are mobilised with a $2 \mathrm{~mm}$ rim of aortic wall, starting at the edge of the transected aorta (fig 4). The proximal $3 \mathrm{~mm}$ of each coronary artery is then mobilised to avoid undue tension, torsion, or kinking of the main artery or one of its branches. The posterior vessel is prepared for coronary anastomosis by making a vertical incision at a right angle to the transected edge (fig 5) at the preselected point. The coronary transfer is completed by anastomosing the rim of the aortic wall around the mobilised ostia to the pulmonary arterial wall, using $6 / 0$ sutures, continuous for half the circumference (fig 6) and interrupted for the remaining part to allow future growth. Before starting the

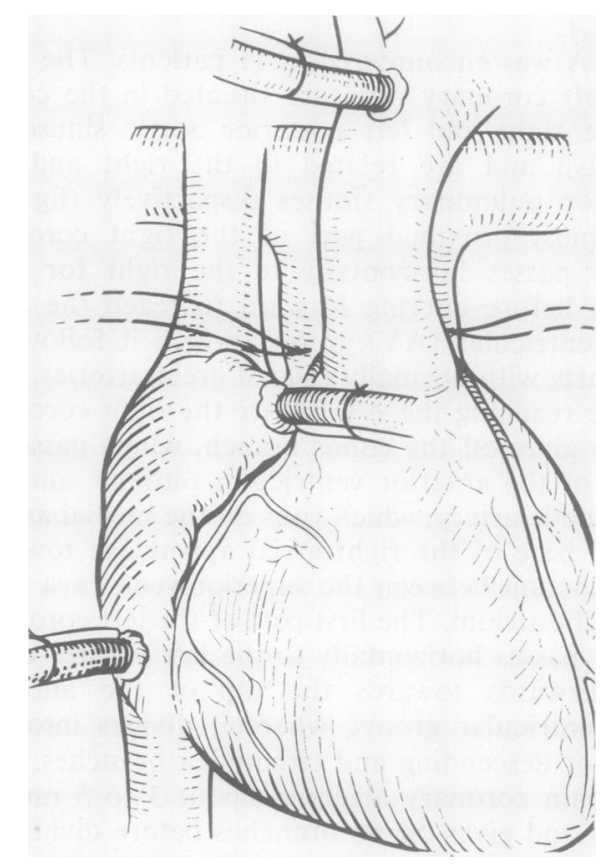

Fig 2 Position of points on posterior vessel for future transfer of coronary ostia (type A) marked by an adventitial stitch.

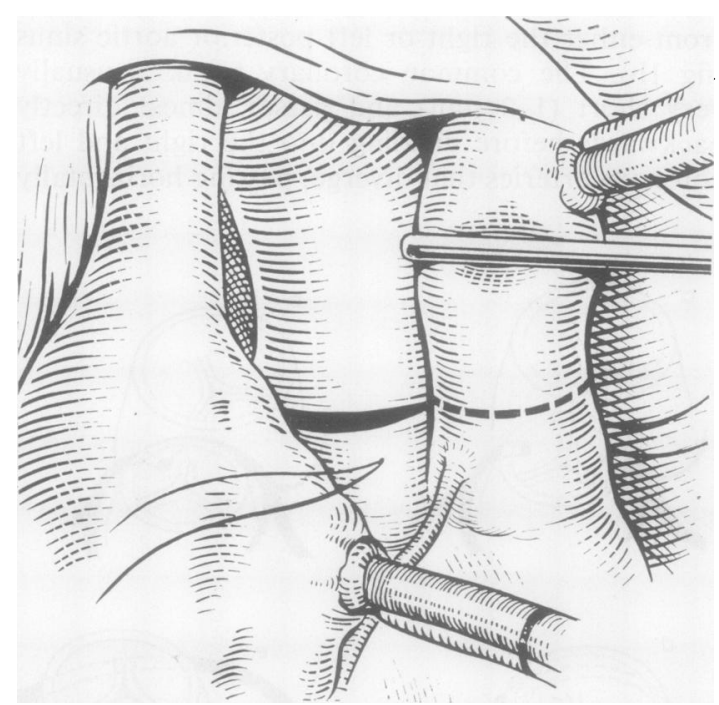

Fig 3 Level of transection of both great arteries.

anastomosis, special care is taken to avoid any axial torsion or kinking of the coronary arteries. The defects in the anterior vessel are repaired 


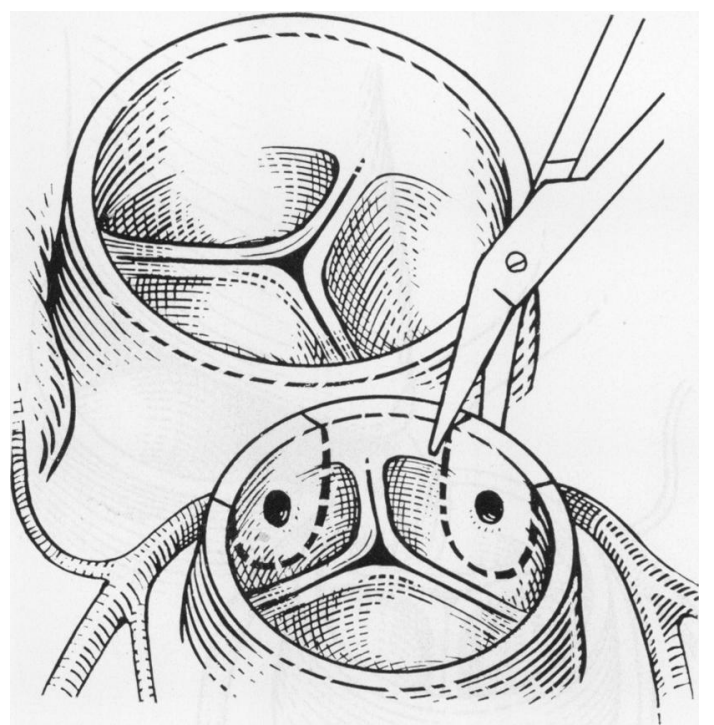

Fig 4 Method of mobilisation of coronary ostia in type $A$.

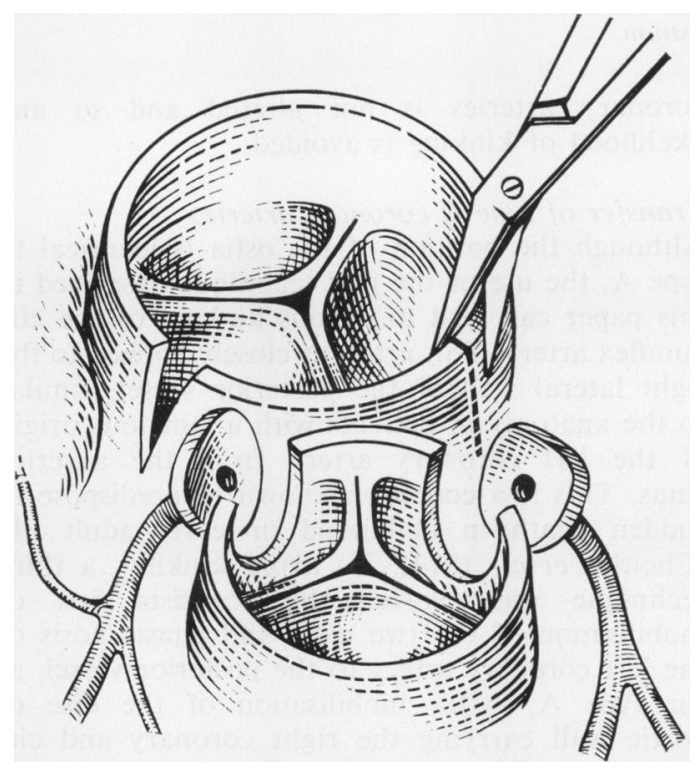

Fig 5 Preparation of posterior vessel for anastomosis of mobilised discs bearing coronary ostia in type $A$.

using homologous dura patches or autogenous pericardium (fig 7).

Transfer of type $B$ and $C$ coronary arteries In patients with type $B$ and $C$ arteries the use of the above-mentioned technique can result in seri-

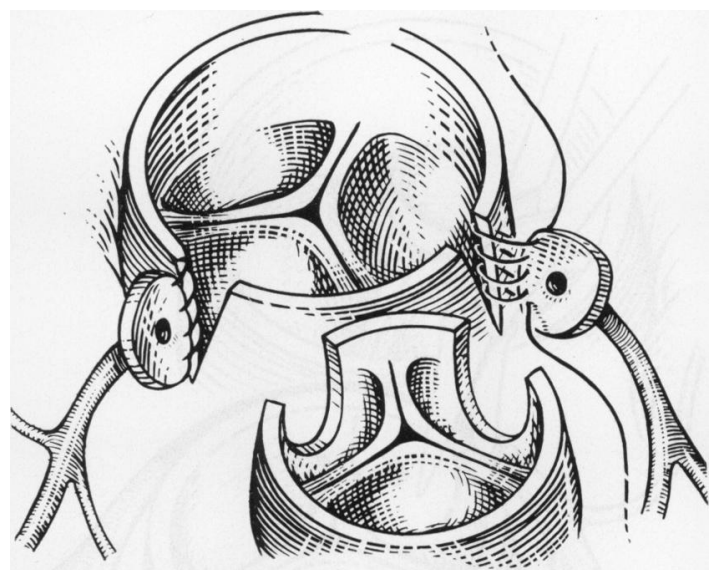

Fig 6 Anastomosis between mobilised coronary ostia and posterior vessel in type A.

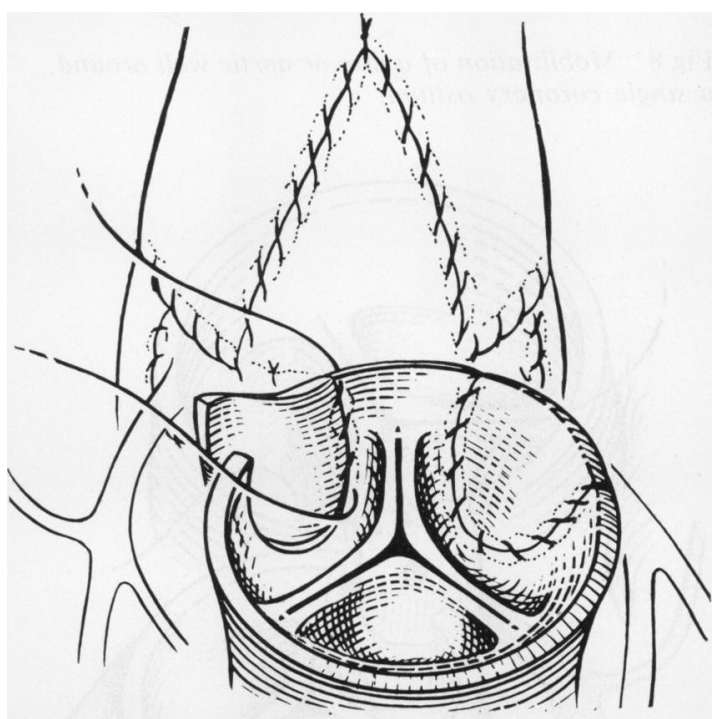

Fig 7 Repair of defects in anterior vessel after transfer of coronary ostia in type $A$.

ous kinking of one or more of the main coronary arteries or their branches. In these patients, after transecting the great arteries, the aortic wall is incised in a semicircular fashion $2 \mathrm{~mm}$ from the rim of the coronary ostium or ostia (fig 8). The resulting disc is mobilised as far as possible. The straight upper border of the rim of aortic wall around the coronary ostium, or ostia, is anastomosed to the adjacent edge of the transected pulmonary artery (fig 9). The distal end of the transected aorta is cut obliquely with a longer anterior "lip." This is anastomosed to the remain- 


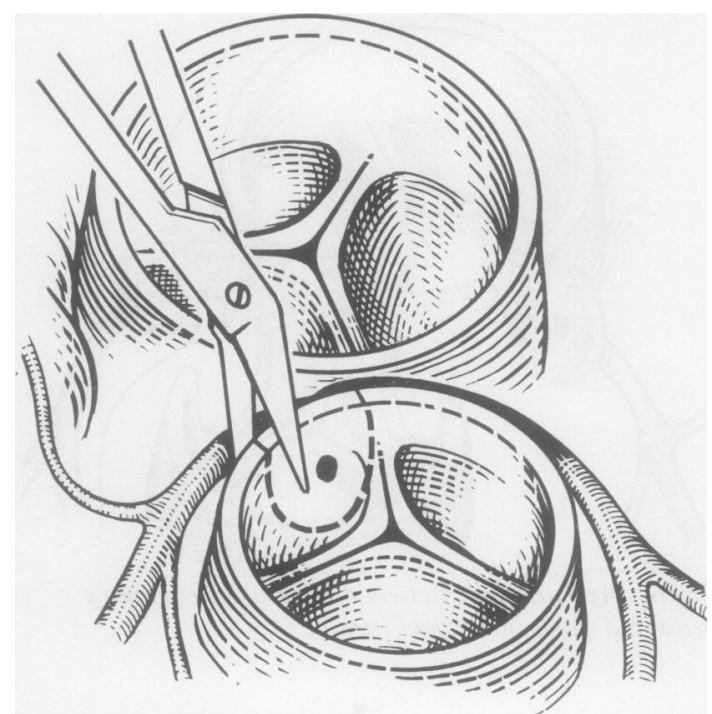

Fig 8 Mobilisation of a disc or aortic wall around a single coronary ostium.

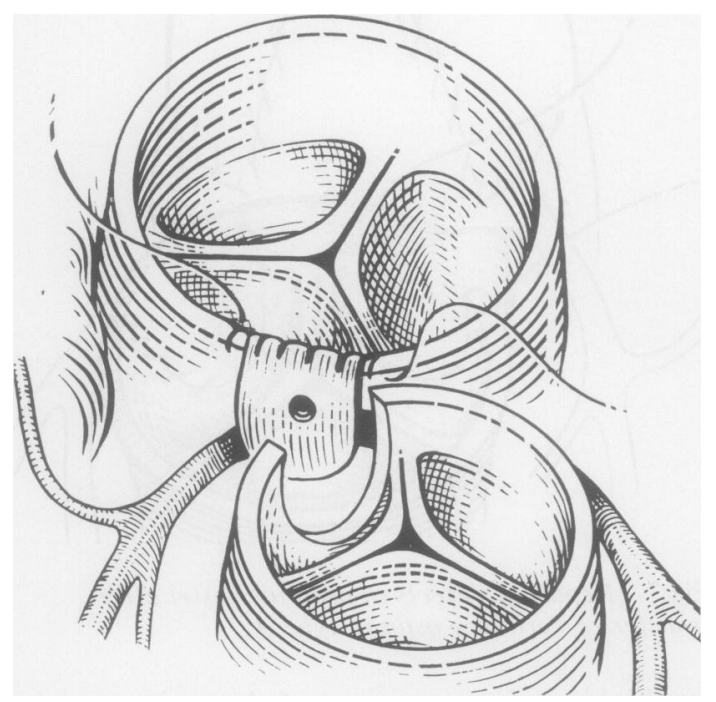

Fig 9 Anastomosis of upper border of mobilised disc bearing a common coronary ostium to border of transected posterior vessel.

ing part of the circumference of the disc around the coronary ostia as part of the anastomosis between the distal aorta and proximal pulmonary artery (fig 10). Thus the coronary arteries arise from a small recess that joins the new aorta with a large opening and has no areas of stasis. The original direction and course of the proximal

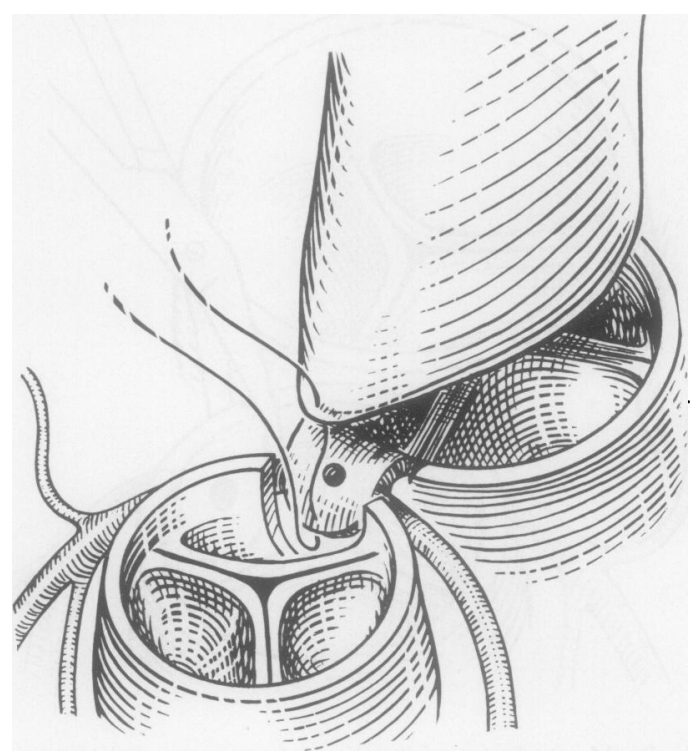

Fig 10 Anastomosis of upper end of transected anterior vessel to lower end of posterior vessel in a fashion to include disc bearing a common coronary ostium.

coronary arteries is not altered and so any likelihood of kinking is avoided.

\section{Transfer of type D coronary arteries}

Although the position of the ostia is identical to type $A$, the use of the first technique described in this paper can lead to serious kinking of the circumflex artery. This artery is closely applied to the right lateral wall of the posterior vessel, similar to the anatomy in patients with anomalous origin of the left coronary artery from the anterior sinus. This is a condition known to predispose to sudden death in childhood or early adult life (Cheitlin et al, 1974). To avoid kinking, a third technique was evolved that consists first of mobilisation of the two ostia and anastomosis of the left coronary artery to the posterior vessel, as for type A. After mobilisation of the disc of aortic wall carrying the right coronary and circumflex arteries, the proximal parts of both of these vessels are mobilised. The posterior margin of the disc of aortic wall is anastomosed to the posterior edge of the incision in the posterior artery (fig 11). A free patch of autologous pulmonary arterial wall is used to join the anterior margin of the disc of aortic wall to the anterior edge of the defect, thus creating a small recess or sinus giving rise to the coronary ostium, which continues to face approximately the same direction 


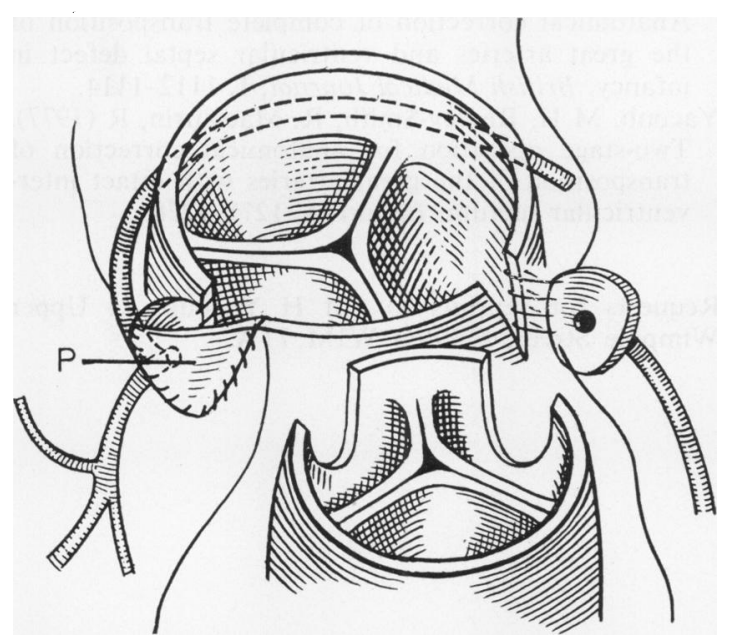

Fig 11 Technique for transfer of type $C$ coronary vessels. To avoid kinking of circumflex artery, a free patch " $P$ " of autogenous pulmonary artery is used to join anterior edge of disc to the anterior edge of defect created in posterior wall.

as before so preventing kinking of the circumflex artery (fig 11). In addition, this technique avoids undue tension on the circumflex artery and its ostium when the future aorta dilates during exercise.

\section{Discussion}

Although the basic abnormality in complete, or d-transposition of the great arteries is the origin of the aorta and coronary arteries from morphological right (anterior) ventricle, and the pulmonary artery from the morphological left (posterior) ventricle, the only available method for treating these patients, until recently, has been by atrial septostomy followed by inflow "correction" by the Mustard or Senning operation (Senning, 1959; Mustard, 1964). These operations have given good early and moderately late results (Breckenbridge et al, 1972), but they are not corrective, as the right ventricle continues to serve the systemic circulation, and there is some concern about the incidence of late arrhythmias (El Said et al, 1972), tricuspid regurgitation (Tynan et al, 1972), and pulmonary or systemic venous obstruction (Stark et al, 1972). The ideal operation, in theory, should consist of transposing the aorta and pulmonary arteries to their appropriate ventricles and resiting the coronary arteries. This corrective procedure is still not widely used. Difficulties in applying this operation are because in patients with intact ventricular septum regres- sion of pulmonary vascular resistance after birth results in rapid regression of left ventricular mass, rendering it incapable of supporting the systemic circulation (Tynan, 1972; British Medical Journal, 1976); added to this are the technical difficulties of transferring the coronary arteries to the posterior vessel at a very young age. It is possible to redevelop the posterior ventricle in patients with intact ventricular septum by performing a first-stage operation consisting of banding the pulmonary artery and creating an aortopulmonary shunt proximal to the band (Yacoub et al, 1977). The technical difficulties of transferring the coronary arteries can be solved only by accurate characterisation of the different anatomical types. We have described five types of coronary artery anatomy and methods for the transfer of each type. These methods are designed to avoid undue tension, torsion, or kinking of the main coronary arteries and their early branches. We hope that the anatomical classification and surgical techniques described here will be of some value in helping to establish the role of anatomical correction in the management of patients with complete transposition of the great arteries.

\section{References}

Breckenbridge, I M, Oelert, H, Stark, J, Graham, G R, Bonham-Carter, R E, and Waterston, D J (1972). Mustard's operation for transposition of the great arteries. A review of 200 cases. Lancet, 1, $1140-1142$.

British Medical Journal (1976). Transposition of the great arteries. British Medical Journal, 1, 11041105.

Cheitlin, M D, De Castro, C M, McAllister, H A (1974). Sudden death as a complication of anomalous left coronary origin from the anterior sinus of Valsalva. Circulation, 50, 780-787.

El Said, G, Rosenberg, H S, Mullins, C E, Hallman, G L, Cooley, D A, and McNamara, D G (1972). Dysrhythmias after Mustard's operation for transposition of the great arteries. American Journal of Cardiology, 30, 526-532.

Jatene, A D, Fontes, V E, Paulista, P P, Souza, L C B, Negaer, F, Galantier, M, Sousa, J E M R (1976). Anatomical correction of transposition of the great vessels. Journal of Thoracic and Cardiovascular Surgery, 72, 364-370.

Mustard, W T (1964). Successful two-stage correction of transposition of the great vessels. Surgery, 55, 469-472.

Ross, D, Rickards, A, Somerville, J (1976). Transposition of the great arteries; logical anatomical arterial correction. British Medical Journal, 1, 11091111.

Senning, A (1959). Surgical correction of transposition 
of the great vessels. Surgery, 45, 966-980.

Stark, J, Tynan, M J, Ashcraft, K W, Aberdeen, E, and Waterston, D J (1972). Obstruction of pulmonary veins and superior vena cava after Mustard's operation for transposition of the great arteries. Circulation, 46, Supp 1, 116-120.

Tynan, M, Aberdeen, E, and Stark, J (1972). Tricuspid incompetence after Mustard's operation for transposition of the greater arteries. Circulation, 45, Supp 1, 111-116.

Yacoub, M H, Radley-Smith, R, Hilton, C J (1976).
Anatomical correction of complete transposition of the great arteries and ventricular septal defect in infancy. British Medical Journal, 1, 1112-1114.

Yacoub, M H, Radley-Smith, R, Maclaurin, R (1977). Two-stage operation for anatomical correction of transposition of the great arteries with intact interventricular septum. Lancet, 1, 1275-1278.

Requests for reprints to: M H Yacoub, 24 Upper Wimpole Street, London W1M 7TA. 